\title{
Proactive information delivery to support eating for older adults with mild and moderate dementia
}

\author{
Luiz Henrique Cavalcanti \\ School of Informatics and Computing - \\ IUPUI \\ Indiana, US \\ luccaval@iupui.edu
}

\author{
Andrew Miller \\ School of Informatics and Computing - \\ IUPUI \\ Indiana, US \\ andrewm@iupui.edu
}

\author{
Richard J Holden \\ Indiana University School of Medicine \\ Indiana, US \\ rjholden@iu.edu
}

\begin{abstract}
Maintaining adequate nutrition for aging populations, especially those affected by dementias such as Alzheimer's disease, is an increasing challenge requiring flexibility for different users and the capacity to adapt as the disease progress. Assistive technologies, such as Proactive Information Delivery (PID), represent a viable approach to assist older adults with dementia in maintaining proper eating practices by monitoring patient routines and context, then providing orientation and prompts that can guide the patient regarding eating related practices decisions. In this poster we present a theoretical overview of the applicability of PID to support persons with mild and moderate dementia with eating. We describe the problem space of eating with dementia, present the concept of PID, and review the literature that supports the applicability of PID to support older adults with mild and moderate dementia to eat properly. Finally, we present and discuss the challenges of adopting Proactive Information Delivery in this sensitive design space, and highlight additional research opportunities that should be further explored.
\end{abstract}

\section{CCS CONCEPTS}

- Applied computing $\rightarrow$ Consumer health • Humancentered computing $\rightarrow$ Interaction paradigms

\section{KEYWORDS}

H.5 - Information interfaces and presentation (HCI); H.5.0 - General.

\section{INTRODUCTION}

With $70 \%$ of the senior population in US living in private homes and the prediction that 1 in 10 older Americans will develop dementia in the next 15 years $[1,2]$, efforts to support patients with Alzheimer's disease and related dementia (ADRD) include non-pharmacological support for patients and their caregivers in daily life activities, especially at home [2,3]. Research has found that patients who experience difficulty eating are more likely to experience problems associated with low food intake (e.g., malnutrition), and that the inability to eat independently is a strong predictor of future institutionalization [4,5]. Empowering patients with ADRD to perform daily activities by themselves can improve their sense of self, resulting in a higher quality of life for both patients and caregivers $[3,4]$.

In this paper we present a theoretical overview to support persons with mild and moderate dementia with eating by the use of Proactive Information Delivery (PID). PID is an interaction paradigm based on the assumption that information systems can identify and anticipate a user's needs and deliver information before the user become aware of it, assuming the role of initial actor $[11,13]$. PID has been explored in specific task-based scenarios where context awareness is relevant and the user is unable to manage all the information relevant to the task[13]. We propose that because of its ability to inform the user without the assumption of previous activation, PID may be a viable approach in this unique and challenging problem space. In the following sections we present the application space of eating with dementia, the concept of PID and the applicability of PID to support older adults with mild and moderate dementia to eat properly. Finally we present and discuss the opportunities and challenges for adopting Proactive Information Delivery and highlight opportunities for research.

\section{EATING WITH DEMENTIA}

Eating difficulty in people with dementia (PWD) is usually associated with reduced cognitive function, which can result in their incapacity to execute cognitive tasks 
involved in the feeding activity [6]. Recent research has shown eating difficulty is related to various cognitive, physical, social, environmental, and cultural factors, all of which people with dementia can be unaware [6,7]. Bunn et al. (2016) identified that interventions impacting a patient's awareness of the food process and eating environment have shown promise in improving food and liquid intake by the participants, especially those patients living in the community [8]. Previous literature also supports that adaptive assistance, or assistance that adjusts to a patient's individual eating ability and context, may produce more positive results in motivating and maintaining adequate eating practices [9].

Watson and Green (2006) have called for more research, quantitative and qualitative, related to interventions to support older adults with dementia with eating properly. Their report highlights that the growing population of older adults who are likely to experience issues with eating is increasing, which is likely to make this issue progressively more critical in the near future [10]. Lack of constant supervision, access to specific products, and knowledge gaps regarding adequate diet and healthy eating habits, can increase the risk of malnutrition and low fluid intake. These risks place individuals at a greater risk for renal, endocrine and cardiovascular problems, which are associated with increased rates of mortality and $[6,8,10]$.

\subsection{Eating challenges for mild and moderate dementia}

Common nutritional concerns associated with PWD, on mild and moderate stages, living at home include safety, weight loss, forgetting or refusing to eat, changes in appetite, dysphagia, and unfavorable eating behaviors [7].

On mild and moderate cases of dementia, the patient is physically capable of performing daily tasks; difficulties of proper eating are related to information access, retention, and attention maintenance [7,10]. Among the impairments impacting eating, we can identify two that are more closely related to information needs: Cognitive Impairment (CI) and perceptual deficits $[2,6]$. While the capacity to retain new information in memory is the most common source of daily issues (frequent asking, losing things, forgetting appointments), the capacity to associate information and make decisions such as what to eat at dinner, which seasoning to use, whether or not to add sugar in coffee and when to drink water, can be more impactful on a patient's quality of life $[1,2]$. In addition, patient condition can be aggravated by perceptual deficit. Differing from CI, this deficit creates a new layer of disorder that impact the comprehension of the information itself, which in combination with CI increases the chances of confusion events and decision making problems $[1,3]$.

To address this complex scenario, assistive technology has to be aware of a user's context and goals, anticipating potential behavior and needs, gathering and providing necessary information in comprehensive format to support the user in their activity without relying on user requests.

\section{PROACTIVITY}

The Cambridge dictionary defines proactive as something (person, Action or event) "intending or intended to produce a good result or avoid a problem." In other words, proactivity is not only related to anticipating future outcomes but guiding the actions that lead to positive results. In HCI, proactivity has been studied primarily with respect to context aware technologies and is closely related to with more established terms as Ubicomp, adaptive interfaces, and AIS.

Ubiquitous and pervasive systems adopt interfaces that range from passive query screens to fully automated status reminders and tools. Recent advances in machine learning techniques, $\mathrm{AI}$ and the access of these systems to larger datasets of data has pushed development to explore preemptive processing and proactive interactions [11,12].

Proactive systems are an alternative interaction approach and direct application of ubiquity and automation with focus on an inverted relationship between technology and user [13]. In a proactive interaction, the technology is responsible for prompting user response. Proactive systems can enhance user's capabilities by highlighting and informing decision moments $[12,13,14]$. Tennenhouse (2000) highlight the need for deeper investigation on how a proactive paradigm of interaction can modify the relationship User-task, once the systems assume the role of supervising data, task and agents [13].

\subsection{Proactive Information Delivery}

A common outcomes of proactive systems is the delivery of context-relevant information for the user. Proactive Information Delivery (PID) assumes that information systems can identify and anticipate user's information needs to deliver the required information, according with defined parameters and preferences, before the user become aware of that need $[11,15,16]$.

To support proactive interaction between systems and user, PID systems should include two characteristics. The first is context-awareness, the second is a decision model that processes data and identifies the information that needs to be presented $[11,16]$. Woerndl, et al. (2011) propose a two-phase proactive context-aware model that produces recommendations for individual users, his model allow information systems to narrow relevant information and deliver it based on context requirements [16].

Unlike reminders and reactive information systems, in PID systems the user is not aware of their lack of information $[12,15,16]$. Proactive systems anticipate and deliver relevant information based on context and 
following the priorities defined by the recommender system [14]. Seibert et al. (2000) explore non-explicit interactions that can happen between users and technology through context [17]. In his work, he highlights that context aware systems can help to minimize interaction complexity, and adjust output format depending on the context.

In an exclusively proactive setting the system will take the first step in identifying specific situations and triggering actions, as proposed by Lei et al. (2007). Their model applies the concept of zero query interactions where the user is not required to provide the initial request for the system [11]. The system would monitor the situation and identify information needs based on multiple sources (sensors, databases, other systems, etc.). Lei's model also includes a personal learning module that maintains and updates individual preferences and behaviors, which are used to feed future tracking interactions.

In summary, PID passively collects information about the user, task, and environment, filtering and analyzing it to identify needs and deliver relevant information in anticipation of user request and/or failure, in order to obtain the best outcome in the current context. A Proactive Information Delivery system will:

- Activate on its own. PID does not require user activation; it will continuously monitor the context and activate itself in defined scenarios and settings.

- Anticipate information needs. The system is capable to identify if the user needs any information/data that can modify his usual behavior or decision process.

- Automate the response or prompt. The system will automatically perform actions to deliver the necessary information to the user.

- Consider users' reactions for future events. With each interaction, the PID system will learn user preferences, and become more accurate and efficient in identifying information relevance and behavioral patterns with time and training.

The system will collect implicit contextual input, and once an information dependency event occurs it will automatically perform an explicit output. Note that an explicit input is not necessary. The user will then perform tasks that will provide additional system input (explicit feedback or implicit changes in context) and will inform the learning module of the system for future interactions.

\section{OPPORTUNITIES AND CHALLENGES FOR PID AND EATING}

The application of PID in open-ended scenarios (to support any kind of task) is limited, but it has potential for applications in dedicated settings where the user does not control all the information or is unaware of specific information needs $[13,15,16]$. Since it requires access to contextual information, it is difficult to apply without controlling or limiting the application environment [13].
This problem makes proactive interfaces more applicable for individual level tasks. Even considering collaboration from external users, the interface will potentially define its recommendation based on an individual profile [12,13,17].

Current research on eating for older adults is focused on the feeding process and more directly on motor control $[14,18,19]$. However, it is necessary to expand investigations on the eating decision process and the underlying causes of eating issues faced by older adults with moderate dementia. Information management and decision making seems to play a major rule in proper eating $[6,8]$ and represents a great opportunity for Proactive Information Delivery systems. The capacity of PID to collect, associate, and suggest can work as a secondary reliable "care-supplier", helping to simplify tasks and reducing cognitive workload [13,19].

O'Leary et al. (2015) and Lopresti et al. (2004) explore the capacity of technology to efficiently track and support patient activities of PWD and supports the fact that independent support technologies can help patients with difficulties in managing and organizing information that is relevant for self-care and health management [18,19]. This is exactly the case of patients with dementia, that can experience memory challenges and other information related difficulties that affect their capacity to perform daily tasks, including maintaining proper eating habits.

PID systems can also support the patient-caregiver relationship, working as an intermediary for recommendations and information exchange, reducing workload and conflict between caregiver and patient [20]. The implementation of PID during initial stages of moderate dementia also has the benefit of providing the system time to learn user preferences and become precise in providing personal relevant information [21]. As the disease progresses, the system will also improve in capacity and the user becomes used to it at the same time, which can lead to a successful intervention that can even follow the patient through later stages of dementia.

A known challenge on adopting PID is that they are task oriented $[13,16]$, and detailed information regarding the eating process and related needs can impact their efficiency and viability. A deeper assessment of the eating related tasks is necessary to correctly implement any assistive intervention with PID.

The use of PID presumes that the user is capable to assimilate the information provided, process it and decide on the actions to take. Designing a system that can support PWD may require the interfaces to be reviewed, in special if the decision maker is not really the patient but the caregiver. While the goal of the intervention is to improve autonomous eating capacity of the patient, the system need to consider that the decision process may include multiple participants and be adaptable to different social scenarios. 


\section{CONCLUSIONS}

Proactive Information Delivery opens doors to new forms of support for context sensitive tasks, and in particular can be an alternative to assist patients with moderate cognitive impairment in proper eating and drinking. Its capacity to coordinate information and simplify the decision making process promises less workload for both patient and caregiver and can reinforce patients' self respect and relationship with the caregiver. Simultaneously, the investigation of Proactive Information delivery systems as an assistive alternative can help us to better understand the differences in relationship between users and PID system, contributing practical data to the HCI community about this still under-explored interaction alternative.

PID can utilize different interaction techniques and does not limit the interface to be used but the information delivery model to be adopted. It is still necessary to investigate the practical information needs associated with the eating process for PWD (from food management and purchase to feeding and discarding food) in order to design and propose an adequate PID intervention for this population. A deeper field investigation may be of great value to inform the design of PID for PWD and can provide initial reference data for future research on eating with dementia.

\section{REFERENCES}

[1] Prince, M., Comas-Herrera, A., Knapp, M., Guerchet, M., \& Karagiannidou, M. (2016). World Alzheimer report 2016: improving healthcare for people living with dementia: coverage, quality and costs now and in the future.

[2] Alzheimer's Association. (2017). 2017 Alzheimer's disease facts and figures. Alzheimer's \& Dementia, 13(4), 325-373.

[3] Giebel, C. M., Sutcliffe, C., Stolt, M., Karlsson, S., Renom-Guiteras, A., Soto, M., ... \& Challis, D. (2014). Deterioration of basic activities of daily living and their impact on quality of life across different cognitive stages of dementia: a European study. International psychogeriatrics, 26(8), 1283-1293.

[4] Lin, L. C., Huang, Y. J., Su, S. G., Watson, R., Tsai, B. W. J., \& Wu, S. C. (2010). Using spaced retrieval and Montessori based activities in improving eating ability for residents with dementia. International journal of geriatric psychiatry, 25(10), 953-959.

[5] Cepoiu-Martin, M., Tam Tham, H., Patten, S., Maxwell, C. J., \& Hogan, D. B. (2016). Predictors of long-term care placement in persons with dementia: a systematic review and metaanalysis. International journal of geriatric psychiatry, 31(11), 11511171.

[6] Priefer, B. A., \& Robbins, J. (1997). Eating changes in mild-stage Alzheimer's disease: a pilot study. Dysphagia, 12(4), 212-221.

[7] Manthorpe, J., \& Watson, R. (2003). Poorly served? Eating and dementia. Journal of advanced nursing, 41(2), 162-169.

[8] Bunn, D. K., Abdelhamid, A., Copley, M., Cowap, V., Dickinson, A., Howe, A., .. \& S Smithard, D. (2016). Effectiveness of interventions to indirectly support food and drink intake in people with dementia: Eating and Drinking Well IN dementiA (EDWINA) systematic review. BMC geriatrics, 16(1), 89.
[9] Lee, K. M., \& Song, J. A. (2015). Factors influencing the degree of eating ability among people with dementia. Journal of clinical nursing, 24(11-12), 1707-1717.

[10] Watson, R., \& Green, S. M. (2006). Feeding and dementia: a systematic literature review. Journal of advanced nursing, 54(1), 8693.

[11] Lei, S., Zhang, K., \& Sha, E. (2007). Applying situational awareness to mobile proactive information delivery. Emerging directions in embedded and ubiquitous computing, 592-603.

[12] Abecker, A., Bernardi, A., Hinkelmann, K., Ku, O. and Sintek, M., 2000. Context-aware, proactive delivery of task-specific information: The knowmore project. Information Systems Frontiers, 2(3-4), pp.253-276.

[13] Tennenhouse, D. (2000). Proactive computing. Communications of the ACM, 43(5), 43-50.

[14] Woerndl, W., Huebner, J., Bader, R., \& Gallego-Vico, D. (2011, October). A model for proactivity in mobile, context-aware recommender systems. In Proceedings of the fifth ACM conference on Recommender systems (pp. 273-276). ACM.

[15] Braunhofer, M., Ricci, F., Lamche, B., \& Wörndl, W. (2015, August). A context-aware model for proactive recommender systems in the tourism domain. In Proceedings of the 17th International Conference on Human-Computer Interaction with Mobile Devices and Services Adjunct (pp. 1070-1075). ACM.

[16] O'Leary, K., Eschler, J., Kendall, L., Vizer, L. M., Ralston, J. D., \& Pratt, W. (2015, April). Understanding Design Tradeoffs for Health Technologies: A Mixed-Methods Approach. In Proceedings of the 33rd Annual ACM Conference on Human Factors in Computing Systems (pp. 4151-4160). ACM.

[17] Seibert, S. E., Kraimer, M. L., \& Crant, J. M. (2001). What do proactive people do? A longitudinal model linking proactive personality and career success. Personnel psychology, 54(4), 845874.

[18] Frank Lopresti, E., Mihailidis, A., \& Kirsch, N. (2004). Assistive technology for cognitive rehabilitation: State of the art. Neuropsychological rehabilitation, 14(1-2), 5-39.

[19] Johansson, L., Björklund, A., Sidenvall, B., \& Christensson, L. (2017). Staff views on how to improve mealtimes for elderly people with dementia living at home. Dementia, 16(7), 835-852.

[20] Kerssens, C., Kumar, R., Adams, A. E., Knott, C. C., Matalenas, L., Sanford, J. A., \& Rogers, W. A. (2015). Personalized technology to support older adults with and without cognitive impairment living at home. American Journal of Alzheimer's Disease \& Other Dementias ${ }^{\circledR}, 30(1)$, 85-97.

[21] Bharucha, A. J., Anand, V., Forlizzi, J., Dew, M. A., Reynolds, C. F., Stevens, S., \& Wactlar, H. (2009). Intelligent assistive technology applications to dementia care: current capabilities, limitations, and future challenges. The American journal of geriatric psychiatry, 17(2), 88-104. 